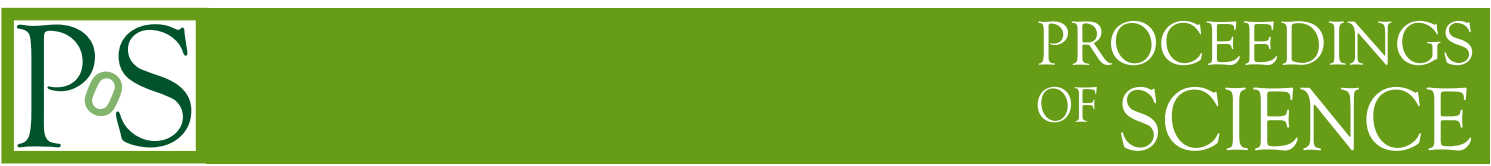

\title{
Recent results from searches for high energy neutrinos with IceCube
}

\author{
Aya Ishihara for the IceCube collaboration* \\ Chiba University, Japan \\ E-mail: aya@hepburn.s.chiba-u.ac.jp
}

The IceCube Neutrino Observatory is a $1 \mathrm{~km}^{3}$ underground Cherenkov detector. The construction of the detector completed in December 2010 at the South Pole. Series of searches for high energy neutrino excesses above the conventional atmospheric neutrino and muon background models have already been performed using data from the partially completed detector.

A hard energy spectrum of cosmic neutrinos from isotropically distributed astrophysical sources could form a detectable signal above the atmospheric background. Recent results from the searches for a diffuse flux of astrophysical neutrinos in data taken between April 2008 and May 2009 with the 40-string configuration of the IceCube detector are presented.

25th Texas Symposium on Relativistic Astrophysics - TEXAS 2010

December 06-10, 2010

Heidelberg, Germany

*Speaker. 


\section{Introduction}

Neutrinos are important probes for exploring the high energy Universe. Because of their unique nature being undeflected in the galactic or extra-galactic magnetic fields and being unabsorbed in the photon filled Universe, neutrinos may fill the missing link between the TeV gammarays and the EeV cosmic-rays. Cosmic neutrinos are expected to be produced by interactions of high energy hadronic beams from cosmic accelerators with surrounding photons and/or matter. However, the neutrino flux produced in each source is highly uncertain. A way of detecting neutrinos from possible weak sources is the diffuse neutrino search. This is because superposition of fluxes from all the weak astrophysical neutrino sources could compose a detectable signal. Still, the expected level of astrophysical neutrino fluxes are so small that it requires a huge target for neutrinos to interact to produce secondary charged particles that can be detected through their Cherenkov emissions. With IceCube, the first cubic-kilometer scale neutrino detector is realized.

The detector construction started in 2005 and was completed in December 2010. IceCube has been in operation with the partial detector configurations since 2005 . IceCube uses $3 \mathrm{~km}$ thick glacial ice as a Cherenkov medium. Cherenkov photons emitted from relativistic charged particles, such as muons and taus, are detected by an array of Digital Optical Modules (DOMs) that amplify and digitally sample the high-speed photomultiplier tube (PMT) pulses. Each DOM encloses a 10" R7081-02 PMT in a transparent pressure sphere along with the high voltage system, a LED flasher board for optical calibration in ice, and a digital readout board. These DOMs are deployed along electrical cable bundles that carry power and information between the DOMs and surface electronics. The cable assemblies, often called strings, were lowered into holes drilled to a depth of $2450 \mathrm{~m}$. The DOMs occupy the last $1000 \mathrm{~m}$ at intervals of $17 \mathrm{~m}$ where the glacial ice is transparent. In addition to the large scale array of optical sensors, the inner dense array called DeepCore which enhances IceCube's performance at low energies was also deployed. DOMs were also frozen into tanks located at the surface near the top of each hole which constitutes an air shower array called IceTop. IceTop provides us with the capability to study the atmospheric muon background reliably. The strings and tanks are arranged in a hexagonal lattice pattern with a spacing of approximately $125 \mathrm{~m}$. The array comprise 5160 DOMs on 86 strings and 320 modules in the surface array as shown in Fig. 1. In 2008-2009, 40 out of 86 strings were deployed and taking data. In this paper, results from the diffuse neutrino searches with the 40 strings configuration of IceCube are presented.

Diffuse neutrino analysis techniques largely differ for energy regions of target neutrinos. This is due to the energy dependence of the neutrino interaction cross-section. The Earth is transparent to neutrinos from all directions up to $\mathrm{PeV}$ energies, where the neutrino mean free paths become significantly smaller than the Earth radius. Thus, extremely high energy neutrinos above $\mathrm{PeV}$ can only enter the IceCube detector from directions above the horizon. The analysis difference also comes from the background spectral shape. Since the conventional atmospheric neutrino and muon spectra are proportional to $E^{-3.7}$ or steeper, and the fluxes steeply decrease with energy. The amount of background events involved in the analyses are significantly different for the energy regions considered. These features led us to the separate diffuse analyses in different energy regions.

In the $\mathrm{TeV}$ region, the neutrinos are searched for using the Earth as the background shield. In this case, the upward-going trajectories are the distinctive feature to separate the neutrino sig- 


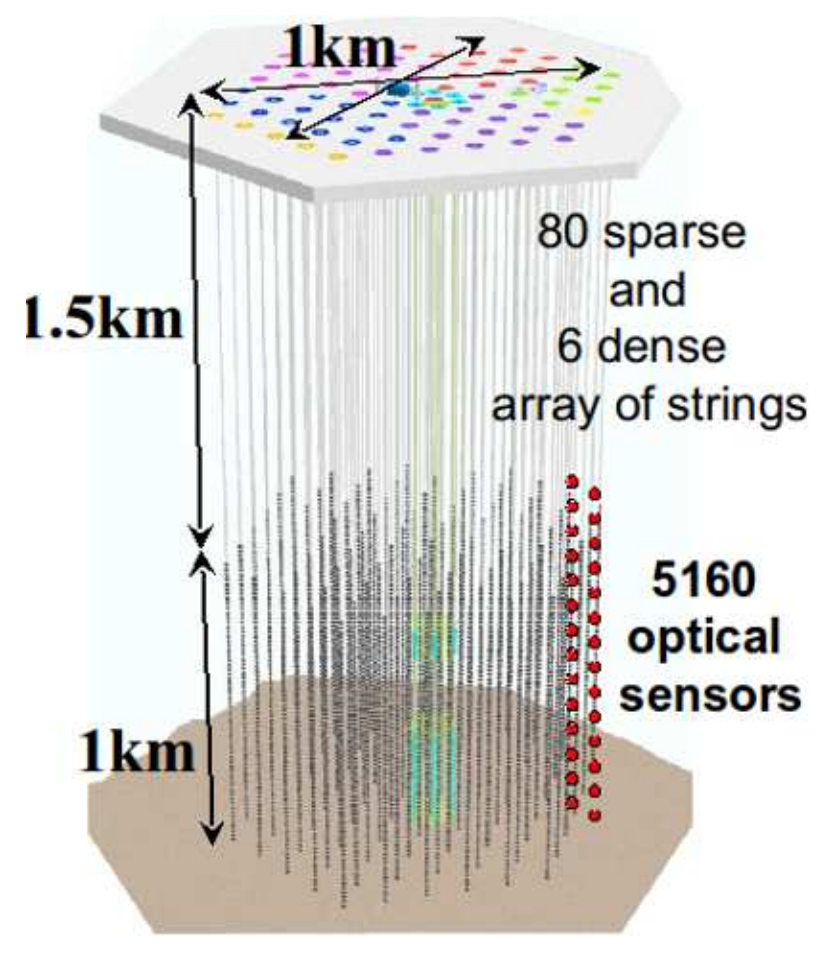

Figure 1: A schematic view of the IceCube detector.

nal from the downward-going atmospheric muon background. Above PeV energies, the primary variable to discriminate signal from background is the energy of the particles. Because the amount of energy deposited in the form of Cherenkov photons by the neutrino-induced charged particles in the detector is highly correlated with their energy, the extremely-high energy neutrino signal stands out against the atmospheric muon and neutrino background due to the much higher light deposition. The total number of photo-electrons (NPE) recorded in an event is used as the main distinctive feature to separate signal from background.

\section{Diffuse Neutrino in TeV-PeV}

Two important steps are required in searches for the $\mathrm{TeV}$ astrophysical diffuse neutrino flux. The first step is the removal of all atmospheric muons from cosmic-ray air showers. This can be achieved by reconstructing the particle direction and rejecting downward-going events. An accurate reconstruction of the particle direction is only possible for elongated muon tracks. Therefore, this selection strategy can only be used to search for muon neutrinos, and other on-going searches for different neutrino flavors have to rely on topological information instead of directional information. The second step is to distinguish possible astrophysical neutrino flux components from the selected upward-going neutrino sample. Possible neutrino sources include potential accelerators of cosmic-rays such as AGNs, SNRs, and GRBs. While astrophysical neutrino flux models involve large uncertainties, many of models predict much harder signal neutrino spectra than that of background atmospheric neutrinos. A possible neutrino component beyond the conventional 


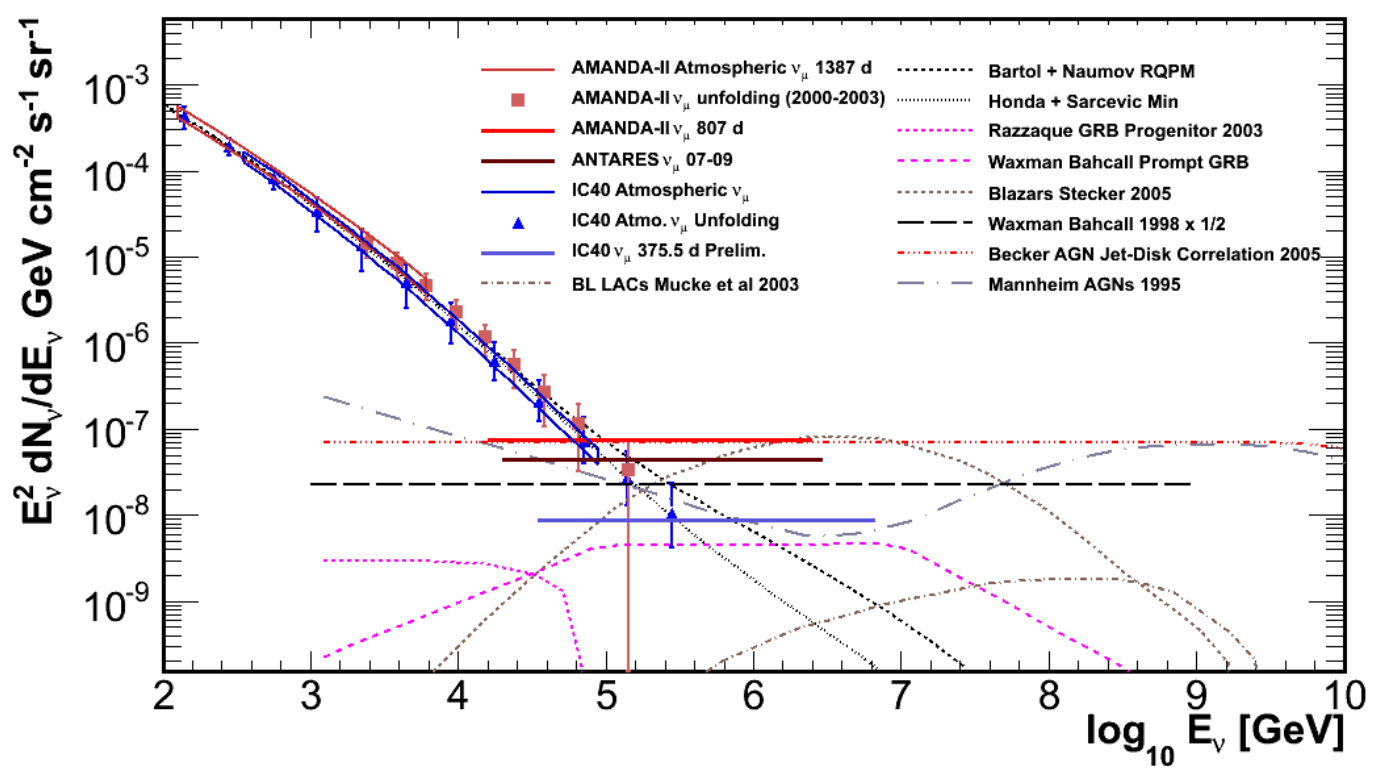

Figure 2: Upper limits on an astrophysical muon neutrino flux with a pure $E^{-2}$ spectrum are shown. Theoretical model predictions of diffuse astronomical muon neutrino fluxes $[1,2,3,4,5,6,7,8,18]$ are also shown for reference along with the other experimental results [9, 10, 11, 20, 21, 22, 25, 24] for comparisons. Model fluxes are that of muon neutrino flavor only, on assumption of standard neutrino oscillations.

atmospheric neutrino flux is searched for in the selected upward-going neutrino sample. The flux and neutrino energy spectrum in this sample is found to be consistent with the conventional atmospheric neutrino models. From the result, an $E^{-2}$ diffuse spectrum integral flux $90 \%$ CL limit is obtained at $E^{2} \phi_{v \mu} \leq 8.9 \times 10^{-9} \mathrm{GeVcm}^{-2} \mathrm{sec}^{-1} \mathrm{sr}^{-1}$ for muon flavor neutrinos in the energy range $3.5 \times 10^{3}-6.9 \times 10^{6} \mathrm{GeV}$. Figure 2 shows the preliminary upper limit to a diffuse flux of neutrinos from extragalactic sources with an $E^{-2}$ neutrino spectrum. The limit probes the region below the Waxman-Bahcall upper bound [3].

\section{Neutrino search: PeV and beyond}

Cosmogenic neutrinos are produced in interactions of the highest energy cosmic-rays with the cosmic-microwave background [13, 14]. They may provide us a direct evidence of the highest energy cosmic ray sources. As already mentioned, the extremely high energy (EHE) neutrino signals were discriminated using the number of photo-electrons (NPE) deposited in the detector. The threshold NPE values are set to a high number $\left(2 \times 10^{6}\right.$ photo-electrons $)$ for the vertically downward-going events while a lower value of $2.5 \times 10^{4}$ photo-electrons is assigned for the events near and below the horizon. This is because the background atmospheric muons are concentrated in the direction of vertically downward-going while expected EHE signals have near horizontal geometries. No signal candidates was found in the data collected in 333.5 days of detector livetime during 2008-2009, consistent with the expected background level of 0.1 events. The quasi- 


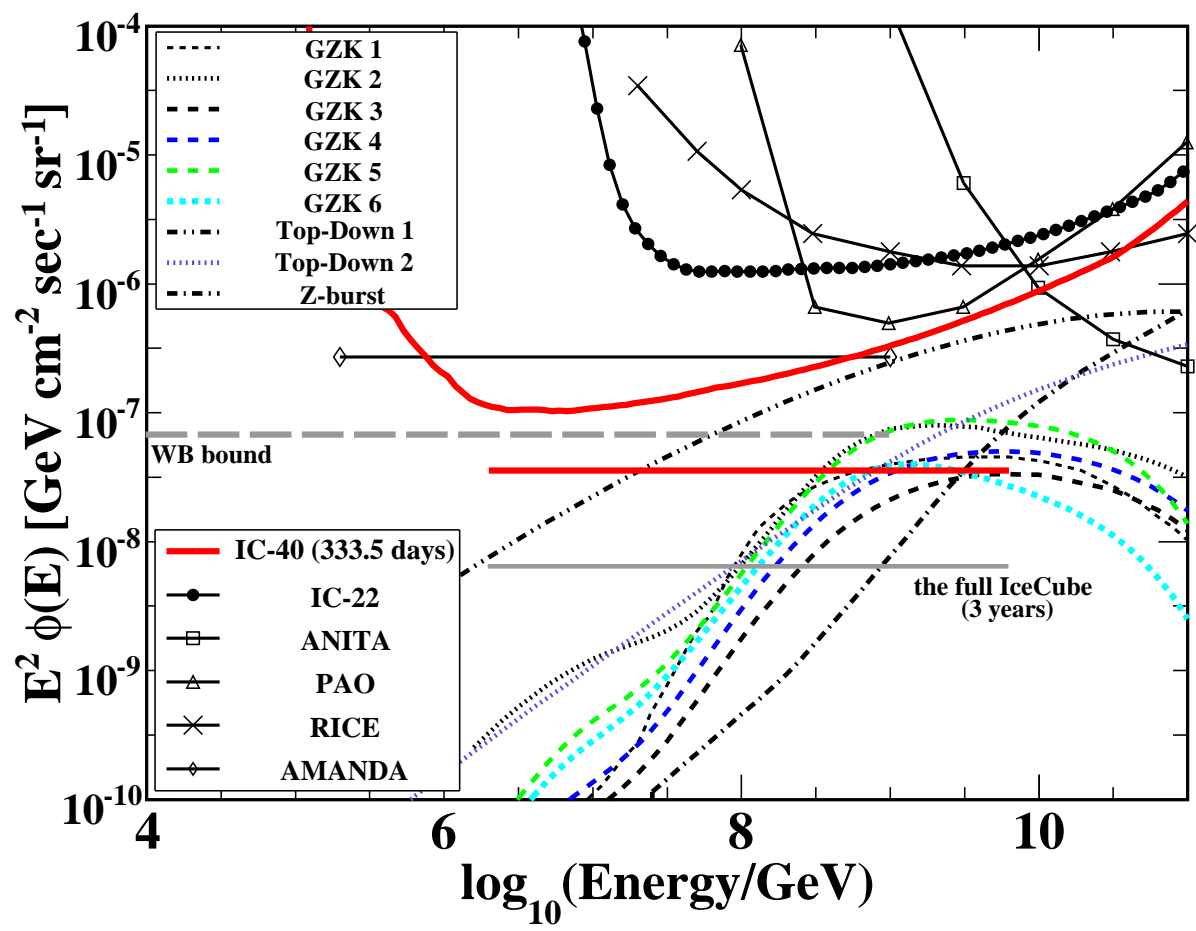

Figure 3: All flavor neutrino flux differential limit and $E^{-2}$ spectrum integrated limit by the 2008-2009 IceCube EHE analysis (red solid lines). The systematic errors are included. Various model predictions are shown for comparison: GZK $1\left(\left(\mathrm{~m}, \mathrm{Z}_{\max }\right)=(4,4)\right)$ [16], GZK 2 [17], GZK $3\left(\Omega_{\Lambda}=0.0\right)$, GZK $4\left(\Omega_{\Lambda}=\right.$ 0.7) [18], GZK 5 (maximal), GZK 6 (the best fit) [19], Z-burst [26], top-down 1 (SUSY) and top-down 2 (no-SUSY) [27]. The gray long-dashed line indicates the Waxman-Bahcall flux bound with cosmological evolution [3]. Model fluxes are summed over all neutrino flavors, on assumption of standard neutrino oscillations. The model independent differential upper limits by other experiments are also shown for Auger (PAO) [21], RICE [22], ANITA [24], and the previous IceCube result (IC22) [12]. Limits from other experiments are converted to the all flavor limit assuming standard neutrino oscillation and a $90 \%$ quasi-differential limit when necessary. The integral flux limit on a pure $E^{-2}$ spectrum is shown for AMANDA-II [25]. For reference, the estimated integrated limit for three years of observation with the full IceCube detector with the same analysis strategy is denoted as a gray solid line.

differential model-independent $90 \%$ CL limit on neutrino fluxes normalized by energy decade is shown in Fig. 3 assuming full mixing in the standard neutrino flavor oscillation scenario. In the limit calculation, the energy decade averaged effective area is used and the contribution from the Glashow resonance [15] is neglected. The $90 \% \mathrm{CL}$ integral flux limit on a pure $E^{-2}$ spectrum obtained at $E^{2} \phi \leq 3.6 \times 10^{-8} \mathrm{GeVcm}^{-2} \mathrm{sec}^{-1} \mathrm{sr}^{-1}$ for cosmic neutrinos with the central $90 \%$ of the $E^{-2}$ signal is found in the energy range $2.0 \times 10^{6}-6.3 \times 10^{9} \mathrm{GeV}$. The limit is also below the Waxman-Bahcall flux bound [3]. These limits are a significantly improved compared to previous results [12]. Expected number of signal events from reference models are also presented in Table 1. 
Table 1: Expected numbers of events in 333.5 days from several cosmogenic neutrino models and top-down models. The confidence interval for exclusion by this observations is also listed when appropriate. Expected event numbers in 3 years of full IceCube operation obtained from a Monte Carlo simulation study are also presented.

\begin{tabular}{lcc} 
Models & $\begin{array}{c}\text { Event Rates (40 strings) } \\
\text { in 333.5days }\end{array}$ & $\begin{array}{c}\text { Event Rates (full detector) } \\
\text { in 3 years }\end{array}$ \\
\hline GZK 1 [16] & 0.57 & 3.1 \\
GZK 2 [17] & $0.91(53.4 \%)$ & 4.9 \\
GZK 3 $\left(\Omega_{\Lambda}=0.0\right)[18]$ & 0.29 & 1.5 \\
GZK 4 $\left(\Omega_{\Lambda}=0.7\right)[18]$ & 0.47 & 2.5 \\
GZK 5 (maximal) [19] & $0.89(52.8 \%)$ & 4.8 \\
GZK 6 (the best fit) [19] & 0.43 & 2.3 \\
Top-down 1 (SUSY) [27] & $1.0(55.7 \%)$ & 5.1 \\
Top-down 2 (no-SUSY) [27] & $5.7(99.6 \%)$ & 31.6 \\
Z-burst [26] & $1.2(66.4 \%)$ & 6.3 \\
WB bound (with evolution) [3] & 4.5 & 24.5 \\
WB bound (without evolution) [3] & 1.0 & 5.5
\end{tabular}

\section{Summary}

The partially completed IceCube detector has already presented a good sensitivity to diffuse neutrino fluxes in wide energy regions. Now the IceCube construction has been finished and the first neutrino detection awaited.

\section{References}

[1] F.W. Stecker, Phys. Rev. D72, 107301 (2005).

[2] A. Mucke et al., Astropart. Phys. 18:593 (2003).

[3] E. Waxman and J. Bahcall, Phys. Rev. D 59023002 (1999); S. Razzaque, P. MÂt'eszaros, and E. Waxman. Phys. Rev. D, 68083001 (2003).

[4] R. Enberg, M.H. Reno, and I. Sarcevic. Phys. Rev. D78, 043005 (2008).

[5] G. Fiorentini, V.A. Naumov, F.L. Villante Phys. Lett. B 510 (2001).

[6] T. Gaisser et al. Phys. Rev. D70, 023006 (2004).

[7] M. Honda et al. Phys. Rev. D75, 043006 (2007).

[8] J.K. Becker, P.L. Biermann, and W. Rhode. Astropart. Phys. 23 (2005) 355-368.

[9] R. Abbasi et al. (IceCube Collaboration), Phys. Rev. D79, 102005 (2009).

[10] R. Abbasi et al. (IceCube Collaboration), Astropart. Phys. 34, 48-58 (2010).

[11] A. Achterberg et al. (IceCube Collaboration), Phys. Rev. D 76, 042008 (2007).

[12] R. Abbasi et al. (IceCube Collaboration), Phys. Rev. D 82, 072003 (2010). 
[13] K. Greisen, Phys. Rev. Lett. 16, 748 (1966);G. T. Zatsepin and V. A. Kuzmin, Pisma Zh. Eksp. Teor. Fiz. 4, 114 (1966) [JETP. Lett. 4, 78 (1966)].

[14] V. S. Berezinsky and G. T. Zatsepin, Phys. Lett. 28B, 423 (1969).

[15] S. L. Glashow, Physical Review 118316 (1960).

[16] S. Yoshida and M. Teshima, Prog. Theor. Phys. 89, 833 (1993); The model with the source evolution $\left(z_{\max }+1\right)^{m}$ with $m=4$ extending to $z_{\max }=4.0$.

[17] O. E. Kalashev, V. A. Kuzmin, D. V. Semikoz, and G. Sigl, Phys. Rev. D 66, 063004 (2002).

[18] R. Engel, D. Seckel, and T. Stanev, Phys. Rev. D 64, 093010 (2001).

[19] M. Ahlers, et al., Astropart. Phys. 34, 106 (2010).

[20] R. U. Abassi et al. (HiRes Collaboration), Astrophys. J. 684, 790 (2008).

[21] J. Abraham et al. (Pierre Auger Collaboration), Phys. Rev. D 79, 102001 (2009). Private communications.

[22] I. Kravchenko et al. (Rice Collaboration), Phys. Rev. D 73082002 (2006).

[23] P. W. Gorham et al. (GLUE Collaboration), Phys. Rev. Lett. 93, 041101 (2004).

[24] P. W. Gorham et al. (ANITA Collaboration), Phys. Rev. D 82, 022004 (2010); arXiv:1011.5004 (erratum).

[25] M. Ackermann et al. (IceCube Collaboration), Astrophys. J. 675, 1014 (2008).

[26] S. Yoshida, G. Sill, and S. Lee, Phys. Rev. Lett. 815505 (1998).

[27] G. Sigl et al., Phys. Rev. Rev. D 59043504 (1998). 